\title{
Calibration of Robertson's Platoon Dispersion Model in Non-lane Based Mixed Traffic Operation
}

\author{
Bhaskar Paul $^{1} \cdot$ Sudeshna Mitra ${ }^{1} \cdot$ Bhargab Maitra $^{1}$
}

Received: 6 November 2015/ Accepted: 18 May 2016/Published online: 1 June 2016

(C) Springer International Publishing Switzerland 2016

\begin{abstract}
Once released from a signalised stop-line, vehicles normally proceed to the next intersection in bunches (called as 'platoon'). While moving along a corridor, vehicle platoons disperses and therefore, the pattern of vehicle arrivals at a signal is different from the pattern of discharge from its upstream signal. Modelling of dispersion of vehicle platoon is an important consideration for coordinated operation of closely spaced traffic signals. In this regard, Robertson's dispersion model has significant contribution towards design of coordinated signal system. However, successful application of Robertson's model largely depends on calibration of the model for local roadway and traffic conditions. In this paper, calibration of Robertson's model is carried out for an urban roadway scenario in India giving due consideration to level of heterogeneity in vehicle mix, variation in travel demand and link travel time. Data are collected from an urban arterial in Kolkata metropolitan area using videography. Using Robertson's platoon dispersion model, downstream arrivals are predicted for observed upstream flow profiles at stop-line. Then, parameters of the Robertson's platoon dispersion model ( $\alpha$ and $\beta$ ) were calibrated using best fit approach. An empirical model is developed to express platoon dispersion factor $(\alpha)$ as a function of vehicle composition, normalised flow (flow to capacity ratio at stop-line), link travel time and modelling step size.
\end{abstract}

Bhaskar Paul

bhaskar_1232@yahoo.com

Sudeshna Mitra

sudeshna@civil.iitkgp.ernet.in

Bhargab Maitra

bhargab@civil.iitkgp.ernet.in

1 Civil Engineering Dept., Indian Institute of Technology, Kharagpur 721302, India
Keywords Robertson's platoon dispersion model · Mixed vehicular stream $\cdot$ Calibration $\cdot$ Empirical model $\cdot$ Link travel time $\cdot$ Vehicle group

\section{Introduction}

Signalised intersections are the integral part of urban roadway system. Traffic operation at signalised intersections greatly influences the overall performance of an urban roadway system. Poor traffic control at signals may contribute to delay, stop, bottleneck and accident. Therefore, rational design of signal timing plan is necessary to improve the efficiency of traffic operation as well as safety on urban corridors. In delay based signal design, green splits are rationally allocated to conflicting traffic movements to achieve a target volume to capacity ratio [1]. However, effective utilisation of available green time is largely influenced by the arrival pattern of vehicles at the stop-line. For an isolated traffic signal, back of queue or queue length which is one of the key performance measures is also affected by the vehicle arrival pattern [2]. Vehicle arrival pattern plays an even more critical role in coordinated operation of closely spaced traffic signals. Non-uniform arrival of vehicles at downstream stop-line affect the utilisation of green split in coordinated phase thus may even cause additional delay to minor road approaches. Hence, characterisation of vehicle arrivals at a signalised stop-line is critical for design of signal timing plan.

Normally, the pattern of vehicle arrivals at a signalised stop-line is distinctively different from the pattern of their discharge from upstream intersection [3]. This is due to mutual interaction of the vehicles, and also their interaction with road side activities. When, vehicles are discharged from an upstream stop-line, they normally move towards 
downstream signal in bunches, each consisting of number of vehicles. These moving bunches are termed as 'vehicle platoon'. Once vehicles are released from the stop-line, they initially move in a tight platoon with short time headways and tend to disperse as travelling further downstream [3-7]. Variation in motorists' desired speed is primarily responsible for dispersion of vehicle platoons [6-8]. Vehicle manoeuvring such as acceleration and deceleration, maintenance of spacing and lane change, vehicle composition, roadway geometry (such as carriageway configuration, grade, curvature [7]), roadside activity (such as parking manoeuvres, pedestrians spillage over carriageway $[8,9]$ ) and prevailing traffic control measures along the traffic corridor are major contributory factors which cause speed variation and thus results, dispersion of vehicle platoons. Due to these, behaviour of platoons changes spatially towards downstream direction. This spatial variation of platoon behaviour is normally studied as 'platoon dispersion'. Apart from signal settings (such as phase sequence) and turning movement pattern at upstream signal, 'platoon dispersion' also influences vehicle arrival profile. Therefore, dispersion of vehicle platoon along a signalised link has been studied by several researchers in the context of signal coordination $[10,11]$.

To model platoon dispersion at signalized intersections, Robertson's recursive model [15] has been used extensively by researchers [5-8]. The rational application of Robertson's model relies on the proper calibration of model parameters, viz. platoon dispersion factor $(\alpha)$ and travel time factor $(\beta)$ [5]. Several studies pertaining to calibration of these two parameters have been reported in the literature for nearly homogenous traffic stream with disciplined lane behaviour [5, 9, 13-18]. However, the traffic stream in developing countries such as India is highly heterogeneous and lane discipline is also absent [19]. The abutting land-use and the level of road side friction prevailing in urban India are distinctly different from those in developed countries. Therefore, the present study aims to calibrate parameters of Robertson's model for a typical signalized traffic corridor in urban India. Accuracy of the Robertson's model also depends on modelling step size [16]. Modelling step size is the duration of time interval over which vehicle discharge counts at stop-line and vehicle arrival counts at downstream sections are aggregated. Therefore, the present study also aims to investigate the effect of modelling step size on prediction error. The study is further extended to development of an empirical model, expressing $\alpha$ as a function of link travel time, vehicle mix and variation in vehicle discharge rate at stop line.

The manuscript is organized in seven sections. In "Literature Review and Motivation", literature review and motivation of the study are discussed in brief, justifying the need of present work. In subsequent Sects. (3, 4, 5 and 6) study methodology, study area and data acquisition, data analysis, result and discussion are demonstrated. Finally, in Sect. 7 major findings of the work are highlighted.

\section{Literature Review and Motivation}

Vehicle platoon behaviour is primarily modelled to predict the arrival of vehicle platoon with time steps and finally to forecast the total arrival time. Size of platoon (i.e., number of vehicles in a platoon), speed of a platoon and distribution of speed within a platoon are the important considerations in modelling of platoon behaviour [20]. Lighthill and Witham's kinematic wave theory [21], Pacey's diffusion model [22] and Robertson's recursive model [12] are some of the major works related to 'platoon dispersion'. These models can predict vehicle arrival profile at a downstream signal for a given discharge profile at upstream signal, and are therefore, used for estimating offset [6] and green split in a coordinated phase.

Lighthill and Witham described the vehicle platoon behaviour using 'kinematic wave theory' [21]. It assumed an equation of continuity to illustrate the changes in traffic state at signalised intersections. Transition between two traffic states was expressed by a shock wave and the speed of a shock wave were estimated by the ratio of changes in flow rate to density [23]. Shock wave characteristics were further used to describe the platoon behaviour along a signalised link. Precise mathematical description of flow state and computational burden-are however identified as the two major concerns of using LWR model [6].

Pacey's diffusion model was built upon pure kinematic theory, which predicts the downstream flow profile with an assumption of normally distributed vehicular speeds in unrestricted overtaking conditions [22]. Dispersion of vehicles in a platoon is assumed to occur due to variation of vehicular speed in platoon. Based on a derived transformed normal distribution of link travel time, Pacey's model estimate the downstream arrivals as a continuous function. In lieu of this, Robertson used a recurrence model to explain platoon dispersion [12]. This model is basically a discrete version of Pacey's diffusion model, which assumed a binomial distribution for vehicle travel time [3]. Out of these three models, Robertson's recursive model has shown satisfactory agreement with field data at under-saturated flow conditions [24] and hence, found effective for traffic signal optimization and performance analysis for a signalized network. The primary advantage of the use of Robertson's dispersion model over Pacey's model is lower computational effort, which is critical for traffic signal optimization in a large study area [3]. Due to ease in rationally applying the Robertson's model for different size 
of traffic network, it has been used vastly around the world [6] and incorporated in number of signal design tools such as TRANSYT-7F [12], Split Cycle Offset Optimization Tool (SCOOT) [25], SATURN [26] and TRAFLO [27].

Robertson's platoon dispersion model is primarily characterised by two parameters; a platoon dispersion factor $(\alpha)$ and a travel time factor $(\beta)$. Mathematical form of the model is given in Eq. 1.

$q_{t}^{d}=F_{n} \times q_{t-T}+\left(1-F_{n}\right) \times q_{t-n}^{d}$

$F_{n}=\frac{1}{1+T_{a} \alpha \beta}$

$T=\beta T_{a}$

where, $q_{t}^{d}$ is arrival flow rate at the downstream signal at time $\mathrm{t}, q_{t-T}^{d}$ is departure flow rate at the upstream signal at time t-T, T is lag time (time gap between initiation of green at upstream stop-line and arrival of first vehicle at downstream stop-line), $T_{a}$ is average link travel time, $\mathrm{n}$ is modelling time step duration, and $F_{n}$ is smoothing factor.

From Eq. 1, it may be inferred that downstream arrivals in each time step are dependent on the departures from an upstream intersection. Downstream arrivals, $q_{t}^{d}$ is a weighted combination of arrival during the previous time step, $q_{t-n}^{d}$ and the departure from upstream intersection $\mathrm{T} \mathrm{s}$ ago, $q_{t-T}$.

Since, Robertson's model estimates the downstream flow at a given time interval, the model needs to be applied recursively to predictthe flow. Seddon $[13,28]$ rewrote the Eq. 1 as,

$q_{t}^{d}=\sum_{i=T}^{\infty} F_{n}\left(1-F_{n}\right)^{i-T} \times q_{t-i+T}$

Equation 4 demonstrates that predicted downstream arrivals follow a shifted geometric series, which estimates the contribution of an upstream flow in $(\mathrm{t}-\mathrm{i})$ th interval to the downstream flow in t-th interval.

As mentioned earlier, accuracy of prediction of Robertson's dispersion model relies on its calibration for local roadway and traffic condition [7]. Improper calibration of $\alpha$ and $\beta$ may result an ineffective signal timing plan [29]. Manar and Bass showed that using a suggested value of $\alpha$ instead of using a locally calibrated value may result in additional delay for an arterial corridor [14]. Due to the significant impact of model parameters $(\alpha, \beta)$ on effective implementation of signal timing plan, several studies were carried out on calibration of these parameters, which could be categorised in two groups. In first group of studies, investigations were carried out to calibrate the model parameters for site specific roadway and traffic condition. In second group of studies, efforts were given to make the calibration procedure more generalised. Basic elements of the dispersion model such as size of modelling time step, and statistical features of travel time were investigated to generalise the calibration framework. A brief discussion on these studies is presented in following section.

In first group of studies, initial investigations were carried out in UK and North America to suggest some default values for model parameters [7]. Subsequently, studies were carried site specific calibration of model parameters. Some of these studies used a fixed value of travel time factor $(\beta)$ to calibrate platoon dispersion factor $(\alpha)$. This is the primary approach of calibration for model parameters which has been used to incorporate $\alpha$ in Transyt-7F signal design tool. In Transyt-7F, platoon dispersion factor $(\alpha)$ is calibrated for a fixed value of $\beta$, which is equal to 0.8 [6]. However, the use of default values of model parameters (viz. $\alpha$ and $\beta$ both or $\beta$ only) may result significant error in modelling of vehicle platoon behaviour [7]. Therefore, studies were also carried out to calibrate both the parameters ( $\alpha$ and $\beta$ ) simultaneously [5]. Majority of these studies used best fit approach to calibrate the Robertson's platoon dispersion model. Significant findings of these studies were briefed by Farzaneh [6] which gave a firsthand impression on variation of model parameters with roadway and traffic conditions. These findings are graphically shown in Fig. 1.
Fig. 1 Best fit parameters of Robertson's model. ${ }^{\text {aPrimarily a }}$ sub-urban arterial corridor with dual carriageway and turning lanes; no parking. ${ }^{\mathrm{b}}$ Typically well designed CBD arterials with light turning traffic, pedestrian traffic, ${ }^{\mathrm{c}} \mathrm{CBD}$ corridors with combination of parking, moderate to heavy turns, pedestrian traffic [6]

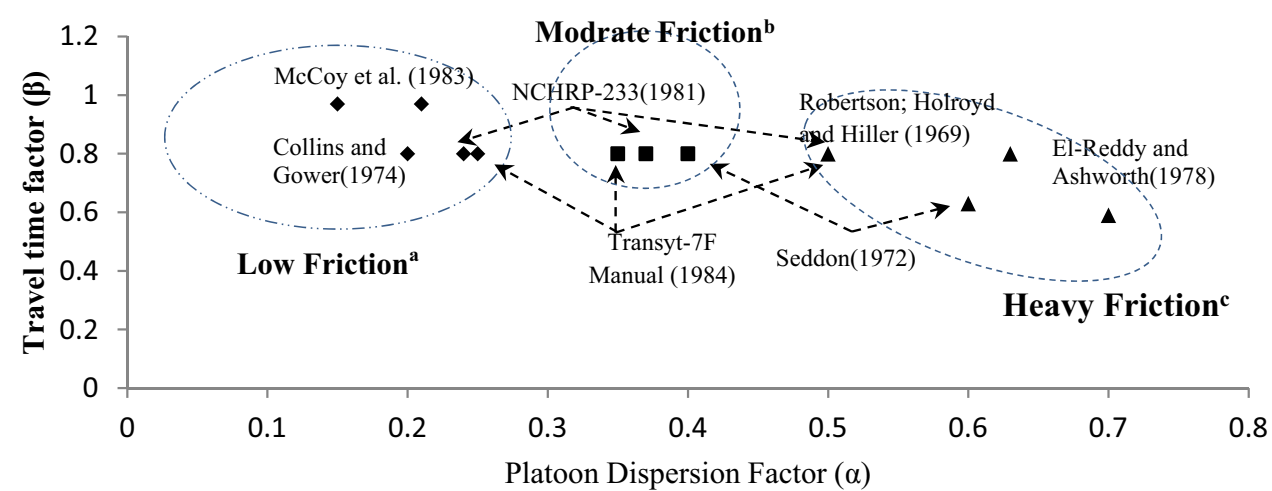


While these studies did not report the effect of major traffic state parameters such as speed, flow and density on platoon dispersion, these parameters are dynamic with respect to time and space and should be included in modelling. To demonstrate this fact, Bass and Lefebvre [15] calibrated platoon dispersion factor based on link travel time and traffic volume. Later, Manar and Bass [14] established a parabolic relation between traffic volumes and platoon dispersion factor $(\alpha)$. It was found that $\alpha$ increases as traffic volume increases and reaches its maximum value when volume becomes half of the roadway capacity. Thereafter, it decreases to a minimum value when traffic volume becomes equal to roadway capacity. However, the relative change between platoon dispersion factor $(\alpha)$ and traffic volume does not follow a unique trend for all carriageway configurations. Therefore, carriageway width has also been identified as an influencing factor of platoon dispersion [8]. To capture the effect of carriageway width on platoon behaviour, Bie et al. [5] developed a set double Gaussian function to relate dispersion factor $(\alpha)$ and normalised flow (volume to capacity ratio at stop-line) for several low friction traffic corridors. Four different corridors were studied with number of vehicle lanes two, three, four and five respectively. Across all carriageway width, platoon dispersion factor $(\alpha)$ was found as high as $0.95-0.98$ when normalised flow was around 0.70-0.75. It was also observed that, dispersion factor $(\alpha)$ changes with normalised flow more rapidly along a wide carriageway than a narrower one.

In second group of studies, investigations were carried out on the functional relations among basic parameters of the Robertson's model such as platoon dispersion factor $(\alpha)$, travel time factor $(\beta)$, smoothening factor $\left(F_{n}\right.$ and inherent travel time distribution. Seddon [13, 28] found that dispersion factor $(\alpha)$ may be expressed as a function of travel time factor ( $\beta$ ) (Eq. 5). Yu and Van Aerde [30] carried out a sensitivity analysis to investigate the effect of travel time distribution on predicted downstream flow profile. It was found that predicted downstream flow profile was not sensitive to any particular distribution of travel time. They proposed numerous equations for calibration based on statistical features of travel time viz. mean $\left(\mathrm{T}_{\mathrm{a}}\right)$ and standard deviation $(\sigma)$. This approach addressed some of the basic limitations of the best fit approach (such as describing the site condition through best fit values of the model parameters) [6]. Use of mean travel time and its standard deviation helped to rationale the effect of site specific factors such as grade, curvature, side friction, traffic volume and other source of impedance on platoon dispersion. Yu [7] proposed following set of equations (Eqs. 6,7 ) to calibrate $\alpha$ and $\beta$. However, this method is more suitable for advanced traffic management system (ATMS) where, travel time data are easily available on real time basis.

$$
\begin{aligned}
\beta= & \frac{1}{1+\alpha} \\
& =\frac{\sqrt{1+4 \sigma^{2}-1}}{2 T_{a}+1-\sqrt{1+4 \sigma^{2}}} \\
F_{n}= & \frac{\sqrt{1+4 \sigma^{2}-1}}{2 \sigma^{2}}
\end{aligned}
$$

The accuracy of the analytical framework is also sensitive to the duration of modelling step size. To address this limitation, Rakha and Farzaneh [16] proposed three enhanced geometric distribution of travel time that overcame the errors due to use of different modelling step size. A sensitivity analysis carried out by Farzaneh and Rakha [17] revealed that performance index (PI) is more sensitive to the travel time factor $\beta$ than platoon dispersion factor $\alpha$ and duration of modelling time step. To overcome the shortcomings of TRANSYT signal design model (which use fixed value of $\beta$ ), a formulation was proposed to control the value of $\beta$ by varying the average link travel time.

Previous discussion clearly indicates that over the years, methodology of application of Robertson's dispersion model has been updated. While initial studies identified the corridor specific roadway and traffic related issues, later, efforts were given to express model parameters (viz. $\alpha$ and $\beta$ ) in relation to variation in travel demand, link travel time (thus putting weightage on link length) and carriageway width with an aim to overcome the limitations of Transyt7F signal design approach. In such context, two major area of research are identified as (1) rational application of Robertson's model through its calibration as per local roadway and traffic condition, and (2) the development of a robust analytical approach for its calibration so as to negate the limitation of Transyt-7F signal design tool.

While most of the past studies on calibration of Robertson's model were carried out on nearly homogenous traffic environment with disciplined lane behaviour, there has been limited work if not any in the context of developing countries such as India where traffic stream is highly heterogeneous with poor lane discipline. In such non-lane based traffic system, driving behaviour of vehicles is influenced by both longitudinal as well as lateral spacing, which has not been investigated in previous studies. As a result, the microscopic behaviours of vehicles such as overtaking and lane changing behaviour observed in lane based traffic cannot be transferrable to non-lane based traffic as they are more likely to be different, which in turn will significantly affect the macroscopic behaviour of vehicles such as platoon dispersion [5]. Additionally, a few past studies [5, 14] have investigated the effect of traffic volume on platoon dispersion factor $(\alpha)$ for lane based traffic system, but the finding may not be again directly applicable for non-lane based heterogeneous traffic as the 
freedom or constraints of manoeuvring in homogeneous traffic stream are likely to be different than that of heterogeneous non-lane based traffic. As a result, there still lies a gap in state of knowledge as to how non-lane based heterogeneous traffic will disperse and how traffic volume and composition affects such dispersion. The present work aims to calibrate the Robertson's dispersion model for a non-lane based traffic system in India and investigate the effects of heterogeneity in vehicle mix and variation in travel demand on vehicle platoon behaviour. Finally, vehicle platoon behaviour is empirically modelled as a function of major traffic state parameters (viz. travel demand, vehicle mix, link travel time).

\section{Study Methodology}

Identification of data requirement and selection of study corridor were the initial steps of the proposed study. Subsequently, the overall work is carried out in five steps namely, development of database, identification of vehicle platoons, selection of modelling step size, calibration of the Robertson's model and development of an empirical model. Brief description of the sub tasks are given in following sections. A typical urban corridor in the Kolkata city is considered for the case study.

\section{Development of Database}

Data were collected from a signalised urban corridor using videography. Cameras were placed at suitable locations to record the vehicle discharge at a signalised sop-line and vehicle arrivals at its three downstream sections located at a distance of 230, 450 and $700 \mathrm{~m}$ from the stop line in order to study the variation of platoon behaviour over length. To capture a wide range of variation in vehicle composition and traffic volume, data collection covered both off-peak and peak hours of a working day.

\section{Identification of Vehicle Platoons}

While departing across the stop-line, vehicles are separated by time headway. Individual vehicle headway generally varies within the range of $0.5-10 \mathrm{~s}$ [27]. In the present study, vehicles separated by larger time headway at stopline are grouped to identify the distinct vehicle platoons. It is assumed that mutual interaction of vehicles within a platoon do not have any effect on its preceding or following vehicle platoon. The threshold value of time headway at stop-line, based on which vehicle platoons are identified is termed as critical headway [4, 31]. With a large critical headway, variances of platoon variables get increased, whereas use of a small critical headway results too many platoons and insufficient platoon information. ElReddy et al. [18] and Bie et al. [5] used $4 \mathrm{~s}$ time interval as critical headway to identify the vehicle platoons at stopline. In the context of present study, which deals with a non-lane based traffic system, distributions of platoon size are studied for three different headways viz. $2 \mathrm{~s}, 3 \mathrm{~s}$ and $4 \mathrm{~s}$ to obtain a suitable value of 'critical headway'. For non-lane based traffic system, headway is defined as the time gap between the two vehicles while passing across the stop-line (weather second one follow the first one or not).

\section{Selection of Modelling Step Size}

Modelling step size is a significant parameter which is critical for calibration of Robertson's dispersion model [16]. Vehicle discharge profile and arrival profile as well, depends on the size of modelling step. With an increase in modelling step size, number of modelling step with zero flow gets reduced. Rakha and Farzaneh [16] adopted three modelling step sizes viz. $2 \mathrm{~s}, 4 \mathrm{~s}$ and $6 \mathrm{~s}$ to investigate the accuracy of the proposed dispersion models. Bie et al. [5] adopted only $4 \mathrm{~s}$ time interval as modelling step size to calibrate $\alpha$. In present study, three modelling step sizes viz. $3 \mathrm{~s}, 4 \mathrm{~s}$ and $6 \mathrm{~s}$ are investigated.

\section{Calibration of Robertson's Dispersions Model}

Downstream arrivals for a given upstream discharge profile is estimated using Eq. 4. To minimise the error between observed arrivals and estimated arrivals, platoon dispersion factor $(\alpha)$ is calibrated for a fixed value of travel time factor ( $\beta$ ) (equals to 0.8 ). For calibration of $\alpha$, following objective function (Eq. 8) is minimised using best fit approach.

$f(\alpha)=\sum_{t=1}^{n}\left[q_{d}^{\prime}(t)-q_{d}^{t}(t)\right]^{2}$

where, $q_{d}^{\prime}(t)$ is observed arrival at downstream section during time step t and $q_{d}^{t}(t)$ is observed arrivals at downstream section during time step t. Minimum value of $f(\alpha)$ is obtained using solver tool (Generalized Reduced Gradient nonlinear method) in Microsoft Excel spread sheet. To investigate the sensitivity of the model error, calibration is carried out for all adopted modelling step sizes viz. $3 \mathrm{~s}, 4 \mathrm{~s}$ and $6 \mathrm{~s}$. Root mean square error (RMSE) (in vehicle/s) is estimated for each modelling step size.

\section{Development of an Empirical Model}

Robertson's dispersion model is originally developed based on the distribution of link travel time and observed lag time. Further research on calibration of the model parameters identified the several other traffic state parameters 
such as traffic volume [15], volume to capacity ratio [5, 14], mean and standard deviation of link travel time [30] as pertinent influencing factors. In present work, an empirical model is developed using non-linear regression technique to express platoon dispersion factor $(\alpha)$ as a function of vehicle composition, normalised flow, link travel time and modelling step size. 'Normalised flow' is defined as the ratio of vehicle discharge rate at stop-line to stop-line capacity.

\section{Study Area and Database}

Based on data requirement and ease of data collection, a 6-lane dual carriageway segment was selected along Kazi Nazrul Islam Sarani in north eastern part of Kolkata city. This traffic corridor is an important arterial connecting NS Bose International Airport to Kolkata metropolitan area and hence, serves high volume of traffic during peak period of the day. A part of this study corridor between Lake Town intersection to Ultadanga intersection was selected for data collection purpose. Road abutting land-use is primarily residential, and therefore impedance due to any roadside activity is largely absent. However, due to presence of two bus stops within the study segment, boarding and alighting by transit users caused low level of impedance to traffic flow. Operating mode of vehicles along the study segment are predominantly passenger car, transit modes (i.e., bus, mini bus), para-transit modes (threewheeler, taxi) and a very negligible share of two-wheeler. The study segment has two foot over-bridges (FOB), which were used to place video cameras at overhead position. Apart stop-line, three downstream sections viz $\mathrm{AA}^{\prime}, \mathrm{BB}^{\prime}$ and $\mathrm{CC}^{\prime}$ were focussed to record the vehicle arrivals. Data were collected during the morning period (6:45 am to 9:45 am) on 25th of August, 2014. Data collection was not extended beyond 9:45 am, as part of the study segment became oversaturated from 9:35 am onwards. Layout of the study segment is shown in Fig. 2. Video files were later processed in the laboratory to manually extract the data. Video files were played simultaneously to track each of the vehicles from the stop-line till it crosses the section $\mathrm{CC}^{\prime}$. Vehicle passing times at each of four sections (i.e., stopline and downstream sections $\mathrm{A}, \mathrm{B}, \mathrm{C}$ ) were noted down with an accuracy of $1.00 \mathrm{~s}$. Data were fed in Microsoft Excel spread sheet and, stored for further analysis.

\section{Analysis, Results and Discussion}

For different critical headway (viz. $2 \mathrm{~s}, 3 \mathrm{~s}$ and $4 \mathrm{~s}$ ) platoons were identified at stop-line. With $2 \mathrm{~s}$ critical headway highest number of platoons (i.e., 227) was identified. Whereas for $3 \mathrm{~s}$ and $4 \mathrm{~s}$ critical headway, number of platoon observed are 106 and 67 respectively. Distribution of platoon size with $2 \mathrm{~s}, 3 \mathrm{~s}$ and $4 \mathrm{~s}$ critical headway are shown in Fig. 3. For $2 \mathrm{~s}$ and $3 \mathrm{~s}$ headway platoon size distribution is highly skewed in left i.e., number of smaller sized platoons are more for $2 \mathrm{~s}$ and $3 \mathrm{~s}$ headway. However, with $4 \mathrm{~s}$ headway, distribution of platoon size is reasonable balanced. Nearly equal shares $(15 \%)$ are observed for platoon size 11-20, 20-30 and 30-40. For platoon size $41-50$, the share is slightly less than $10 \%$. Share of very small sized platoon (1-10) and large sized platoon (61-60, $71-80,81-90)$ are significantly low (less than $3 \%$ ). Therefore, with $4 \mathrm{~s}$ headway, platoon size distribution was reasonable enough to give adequate platoons information and hence, $4 \mathrm{~s}$ is selected as threshold value of critical headway. Identified vehicle platoons were further analysed to measure the dispersion at different downstream sections viz. section $\mathrm{AA}^{\prime}, \mathrm{BB}^{\prime}$ and $\mathrm{CC}^{\prime}$.

Calibrated $\alpha$ for three downstream sections ( $\mathrm{AA}^{\prime}, \mathrm{BB}^{\prime}$ and $\mathrm{CC}^{\prime}$ ) are henceforth denoted as $\alpha_{230}, \alpha_{450}$ and $\alpha_{700}$ respectively. With $95 \%$ confidence interval, variation of $\alpha_{230}, \alpha_{450}$ and $\alpha_{700}$ are shown in Fig. 4. These variations at each section may be attributed to variation in travel

Fig. 2 Layout of the study section

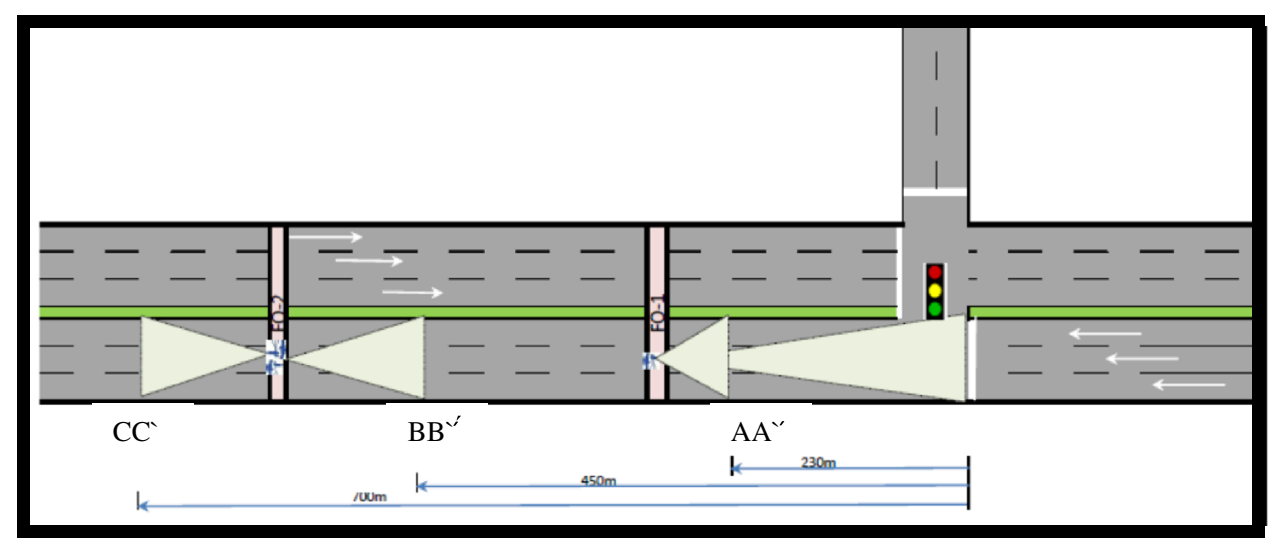


Fig. 3 Distribution of platoon size with 2,3 and $4 \mathrm{~s}$ headway

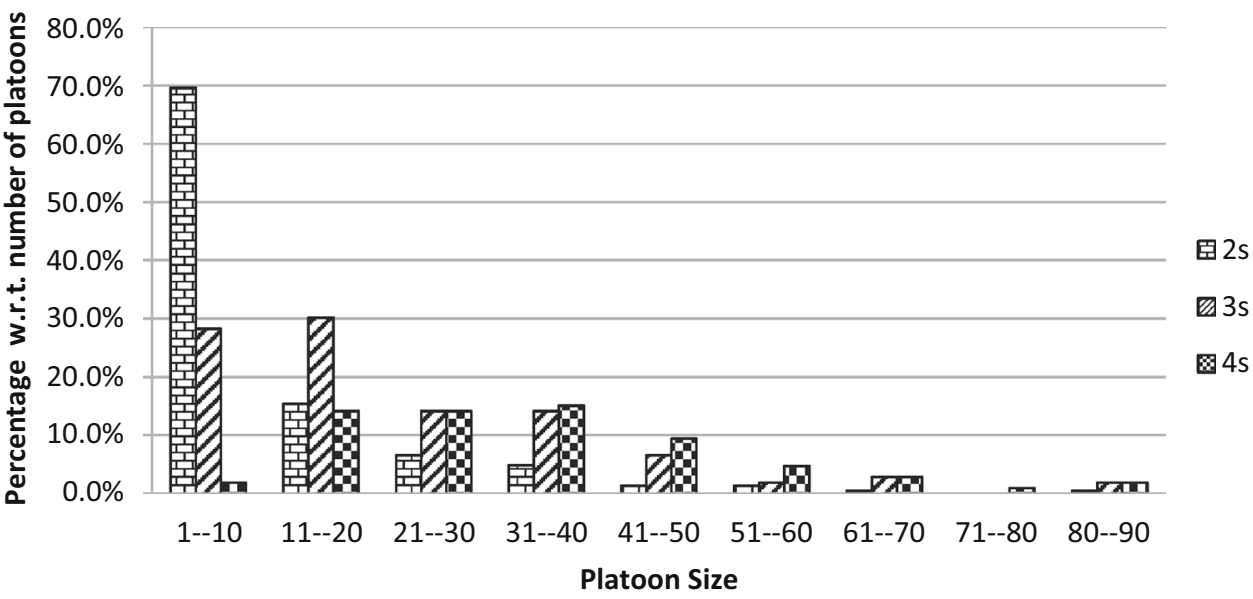

Fig. 4 Variation of $\alpha_{230}, \alpha_{450}$ and $\alpha_{700}$ with different modelling step size

Fig. 5 RMSE for different modelling step size

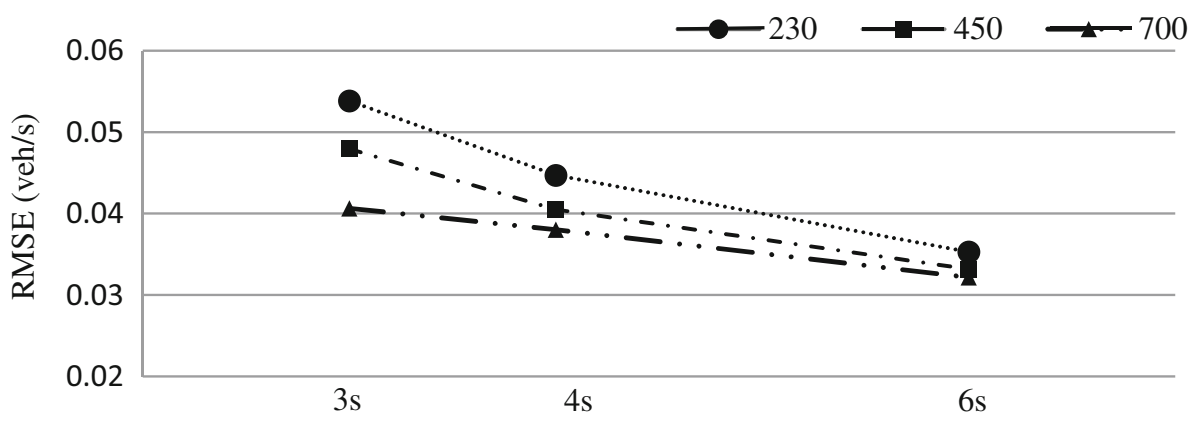

Modeling Step Size

demand and heterogeneity in vehicle mix. Figure 4 depicts that with the increase in modelling step size, $\alpha$ get decreased at each downstream section. For instance, with $3 \mathrm{~s}$ modelling step size, $\alpha_{230}$ varies from 0.11 to 0.24 . However, for $4 \mathrm{~s}$ and $6 \mathrm{~s}$ modelling step size, the variation of $\alpha_{230}$ is observed within the range of $0.07-0.21$ and 0.04-0.17 respectively. Similar trends were also observed for $\alpha_{450}$ and $\alpha_{700}$. Figure 4 also represents that over different modelling step sizes $\alpha_{230}$ is relatively higher than $\alpha_{450}$ and $\alpha_{700}$. However, $\alpha_{450}$ and $\alpha_{700}$ are found at similar level. Average root mean square error for $\alpha_{230}, \alpha_{450}$ and $\alpha_{700}$ are shown in Fig. 5. Figure 5 represents a decrease in RMSE with the increase in modelling step size. This is quite rational as use of large modelling time step in place of smaller time step reduces the numbers of 'time step with zero flow' and, thereby decreasing the scale of prediction error by the model. This is illustrated through Fig. 6a-c. In Fig. 6a, vehicle discharge profiles at stop-line 
Fig. 6 a Observed vehicle discharge profile at stop-line with 3 and 4 s time step. b Observed and predicted arrivals at section $\mathrm{AA}^{\prime}$ with $3 \mathrm{~s}$ time step. c Observed and predicted arrivals at section $\mathrm{AA}^{\prime}$ with 4 s time step
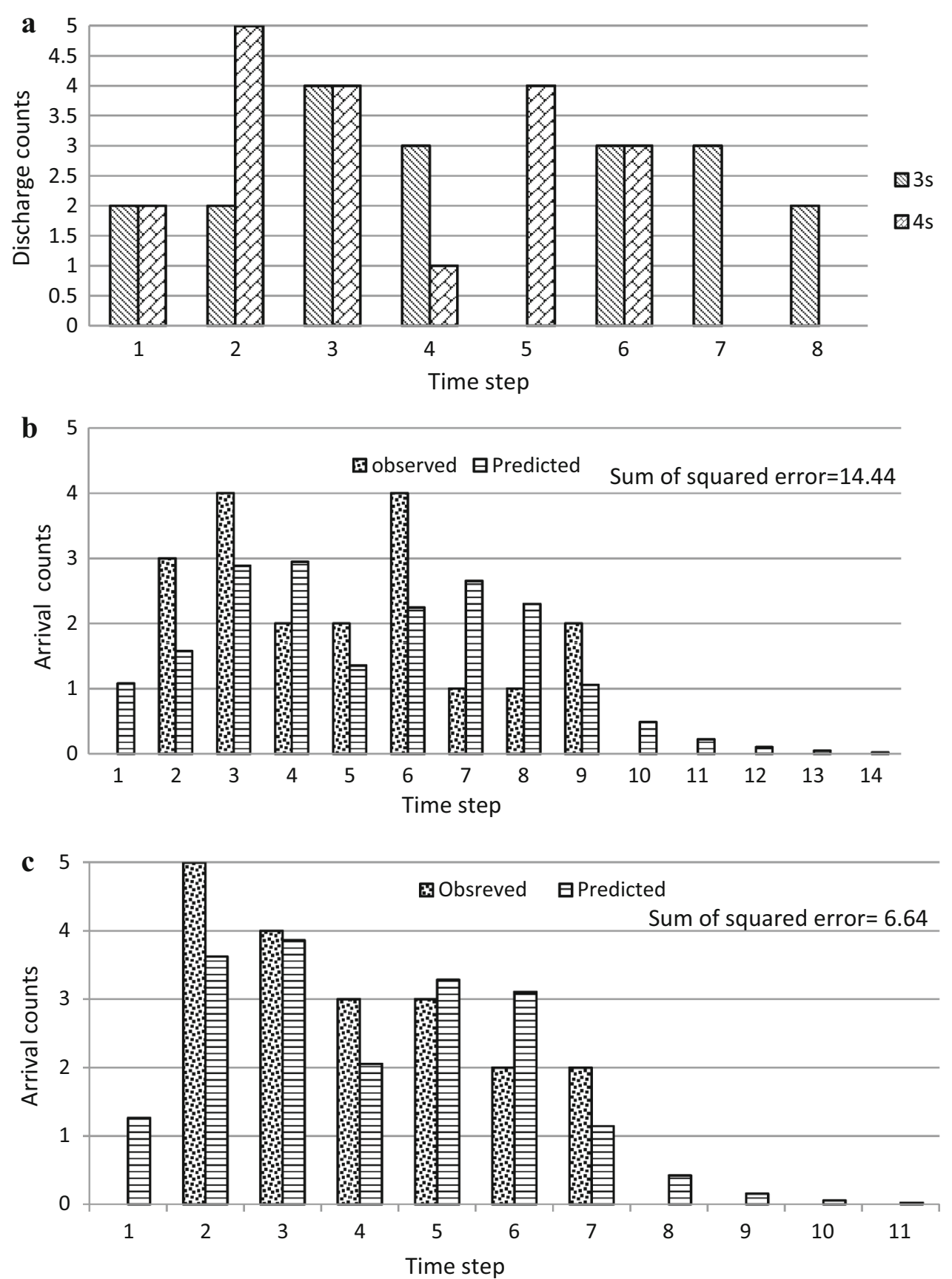

with $3 \mathrm{~s}$ and $4 \mathrm{~s}$ time step are shown. For $3 \mathrm{~s}$ time step, fifth discharge count is 'zero', whereas for $4 \mathrm{~s}$ time step, it is added to the discharge count of earlier time step, thus resulting a profile without having any 'time step with zero flow'. In Fig. 6b, c, observed and predicted downstream arrivals (at section $\mathrm{AA}^{\prime}$ ) of the same platoon is shown for $3 \mathrm{~s}$ and $4 \mathrm{~s}$ time step respectively. From Fig. 6b, c, it may be found that with increase in modelling step size i.e., from $3 \mathrm{~s}$ to $4 \mathrm{~s}$, sum of squared errors has reduced from 14.44 to 6.44. Similar trend has also been reported by Rakha and Farzaneh [16]. It may also be noted that use of large modelling time step in place of smaller time step, accumulates more vehicles in each time step resulting a gross increase in rate of downstream arrivals i.e., less dispersion in vehicle arrival. Therefore, $\alpha$ is found to decrease with an increase in modelling step size.

\section{Model Development}

Platoon dispersion factor $(\alpha)$ are not found to be fixed for different modelling step sizes nor for all three downstream sections (as shown in Fig. 4).Variations in $\alpha$ are also observed in the context of normalised flow, vehicle composition and link travel time. In this regard, formulation of an empirical 
Fig. 7 Distribution of observed peak discharge rate at study stop-line

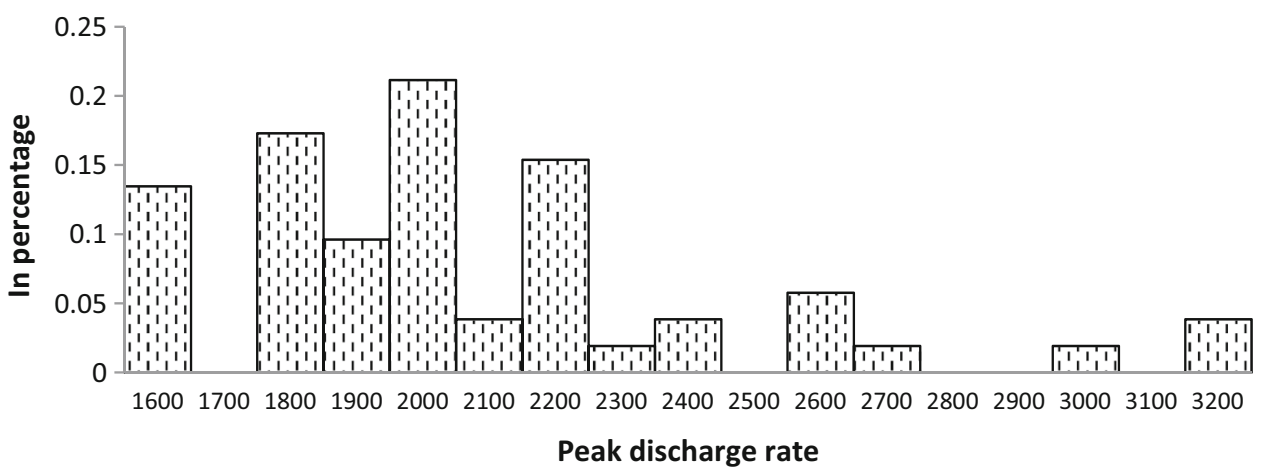

$v_{i}=\frac{q_{i}}{t_{h i}-t_{t i}} \times 3600$

where, $v_{i}$ is discharge flow rate of platoon at stop-line (pcu/h), $q_{i}$ is estimated number of vehicles (in pcu) in platoon $i, t_{h i}$ is time point at which front axle of the leading vehicle of platoon $i$ move across the stop-line, $t_{t i}$ is time point at which front axle of the tail vehicle of platoon $i$ move across the stop-line.

Stop-line capacity may be inferred as the saturation flow rate of the signalised approach. Available guidelines [2, 32] do not address the issues of vehicle driving behaviour at stop-line for a non-lane based traffic system. Therefore, for estimation of stop-line capacity (s), peak discharge rates at stop-line are analysed for the signalised approach. Distribution of observed 'peak discharge rate' is shown in Fig. 7. For different observations, 'peak discharge rate' is found to vary in the range of $1600-3200 \mathrm{pcu} / \mathrm{h} / \mathrm{lane}$. For majority of the observations $(66 \%)$, peak discharge rate falls within $1800-2200 \mathrm{pcu} / \mathrm{h} / \mathrm{lane}$. However, highest frequency was found against $2000 \mathrm{pcu} / \mathrm{h} / \mathrm{lane}$. Therefore, stop-line capacity was estimated as $3 \times 2000=6000 \mathrm{pcu} / \mathrm{h}$. Share of observed platoons at different level of normalised flow $(v / s)$ are given in Fig. 8.

\section{Vehicle composition}

Vehicle composition of the traffic stream, as observed during the study period is given in Fig. 9. To capture the
Fig. 9 Observed vehicle composition at different hours of the study period
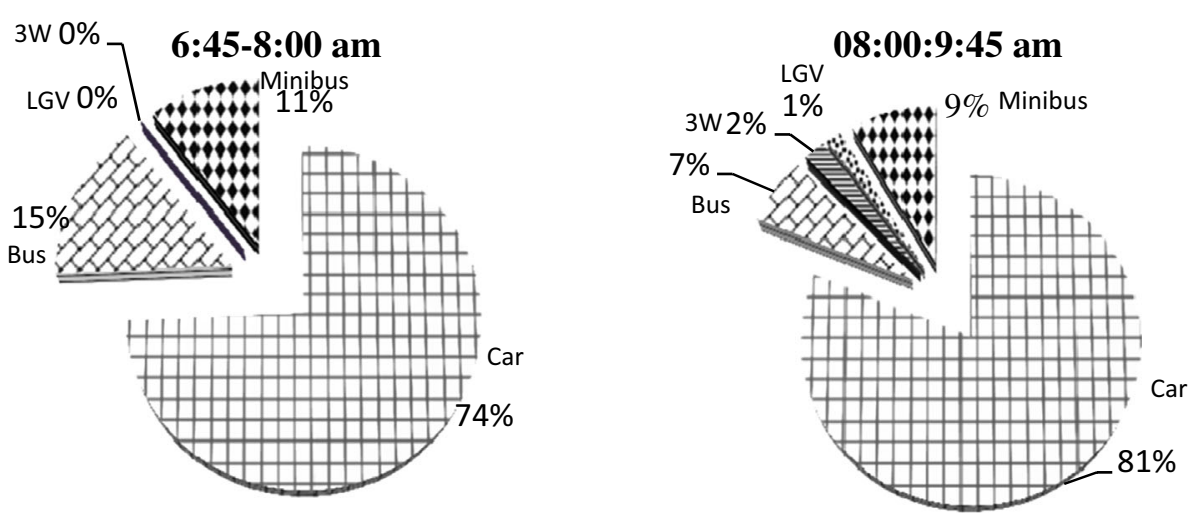


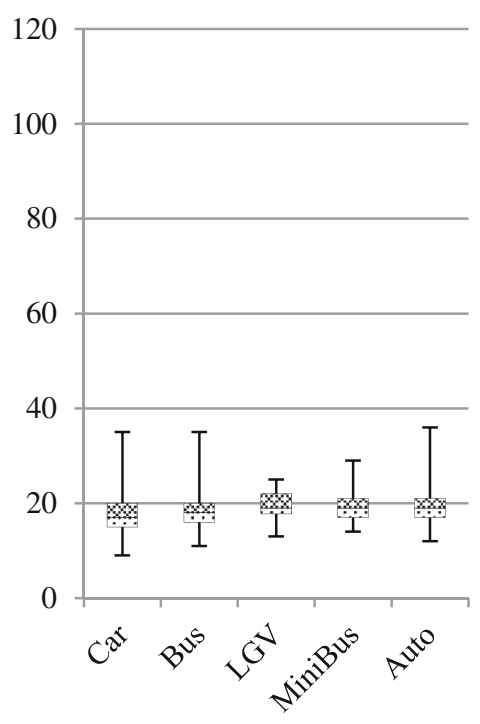

At section AA

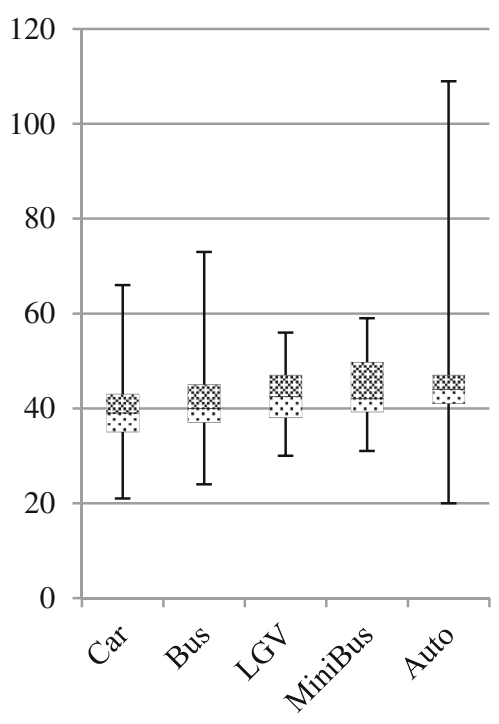

At section $\mathrm{BB}$

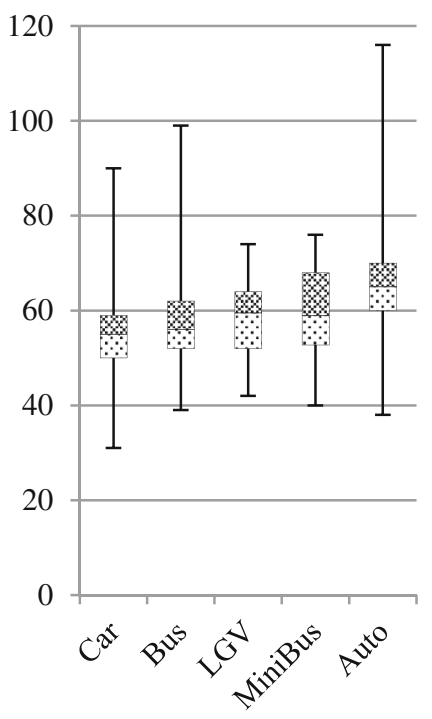

At Section CC

Fig. 10 Box plot of travel time for different vehicular modes

Table 1 Results of statistical tests for travel time of bus, mini bus and LGV

\begin{tabular}{|c|c|c|c|c|c|c|c|c|}
\hline \multirow[t]{2}{*}{ Downstream section } & \multicolumn{3}{|c|}{ Sample size } & \multirow[t]{2}{*}{ Vehicle mode tested } & \multicolumn{2}{|c|}{ F test } & \multicolumn{2}{|l|}{$\mathrm{t}$ test } \\
\hline & Bus & Mini bus & LGV & & $\mathrm{F}_{\mathrm{c}}$ & $\mathrm{f}$ & $\mathrm{t}_{\mathrm{c}}$ & $\mathrm{t}$ \\
\hline \multirow[t]{3}{*}{$\mathrm{AA}^{\prime}$} & 207 & 37 & 26 & Bus and minibus & 1.58 & 1.19 & 1.97 & -1.38 \\
\hline & & & & Bus and LGV & 1.74 & 1.35 & & -1.06 \\
\hline & & & & Mini bus and LGV & 1.88 & 1.16 & & 0.11 \\
\hline \multirow[t]{3}{*}{$\mathrm{BB}^{\prime}$} & 195 & 36 & 26 & Bus and minibus & 1.59 & 1.07 & 1.97 & -1.19 \\
\hline & & & & Bus and LGV & 1.74 & 1.32 & & -0.48 \\
\hline & & & & Mini bus and LGV & 1.89 & 1.23 & & 0.48 \\
\hline \multirow[t]{3}{*}{$\mathrm{CC}^{\prime}$} & 194 & 34 & 26 & Bus and minibus & 1.62 & 1.09 & 1.97 & -0.92 \\
\hline & & & & Bus and LGV & 1.74 & 1.65 & & -0.27 \\
\hline & & & & Mini bus and LGV & 1.90 & 1.51 & & 0.49 \\
\hline
\end{tabular}

effect of vehicle composition on $\alpha$, observed vehicular modes were categorised in three vehicle groups namely Group I: Car, Group II: Bus, minibus and LGV, and Group III: 3 wheeler. These vehicle groups are henceforth referred as $V G-I, V G-I I$ and $V G-I I I$ respectively. Box plot of travel time for different vehicular modes are shown in Fig. 10. Statistical tests ( $F$-test and $t$ test) were carried out with a null hypothesis that travel time for bus, minibus and LGVs in $V G-I I$ are not statistically significantly different. Table 1 shows the result of F-test and t-test. Based on the results, null hypothesis cannot be rejected i.e., grouping of bus, minibus and LGVs is rational. Similar test were repeated to determine whether travel time of different vehicle groups viz. $V G-I, V G-I I$ and $V G-I I I$ are significantly different or not. The null hypothesis assumed that travel time for all vehicle groups have no difference with a given significance level $(p)$ of 0.95 . Table 2 shows the result of these statistical tests. Based on the results, null hypothesis can be rejected i.e., categorisation of vehicle groups are justified.

\section{Link Travel Time $\left(t_{\text {mean }}\right)$}

Distributions of link travel time for different platoons are shown in Fig. 11. For downstream sections $\mathrm{BB}^{\prime}$ and $\mathrm{CC}^{\prime}$ variations in link travel time are larger than section $\mathrm{AA}^{\prime}$ i.e., range of platoon dispersion is more towards faraway sections than stop-line adjacent section.

\section{Proposed Model}

The variation of platoon dispersion factor $(\alpha)$ with the chosen variables (viz. link travel time, modelling step size, vehicle composition and normalised flow) were studied to infer the empirical relation of these variables 
Table 2 Results of statistical tests for travel time of $V G-I$, $V G-I I$ and $V G-I I I$

\begin{tabular}{|c|c|c|c|c|c|c|c|c|}
\hline \multirow[t]{2}{*}{ Downstream section } & \multicolumn{3}{|c|}{ Sample size } & \multirow[t]{2}{*}{ Parameters considered } & \multicolumn{2}{|c|}{ F test } & \multicolumn{2}{|l|}{$\mathrm{t}$ test } \\
\hline & VG-I & VG-II & VG-III & & $\mathrm{F}_{\mathrm{c}}$ & $\mathrm{f}$ & $t_{c}$ & $\mathrm{t}$ \\
\hline \multirow[t]{3}{*}{$\mathrm{AA}^{\prime}$} & 1762 & 270 & 241 & $V G-I$ and $V G-I I$ & 0.86 & 0.89 & 1.96 & -5.06 \\
\hline & & & & $V G-I$ and $V G-I I I$ & 1.22 & 1.14 & & 9.54 \\
\hline & & & & $V G-I I$ and $V G-I I I$ & 0.81 & 0.37 & & 11.45 \\
\hline \multirow[t]{3}{*}{$\mathrm{BB}^{\prime}$} & 1760 & 257 & 232 & $V G-I$ and $V G-I I$ & 0.86 & 0.63 & 1.96 & -7.49 \\
\hline & & & & $V G-I$ and $V G-I I I$ & 0.85 & 0.55 & & -13.7 \\
\hline & & & & $V G-I I$ and $V G-I I I$ & 0.80 & 0.87 & & -4.20 \\
\hline \multirow[t]{3}{*}{$\mathrm{CC}^{\prime}$} & 1750 & 254 & 219 & $V G-I$ and $V G-I I$ & 0.86 & 0.67 & 1.96 & -0.92 \\
\hline & & & & $V G-I$ and $V G-I I I$ & 0.85 & 0.51 & & -0.27 \\
\hline & & & & $V G-I I$ and $V G-I I I$ & 0.80 & 0.77 & & 0.49 \\
\hline
\end{tabular}

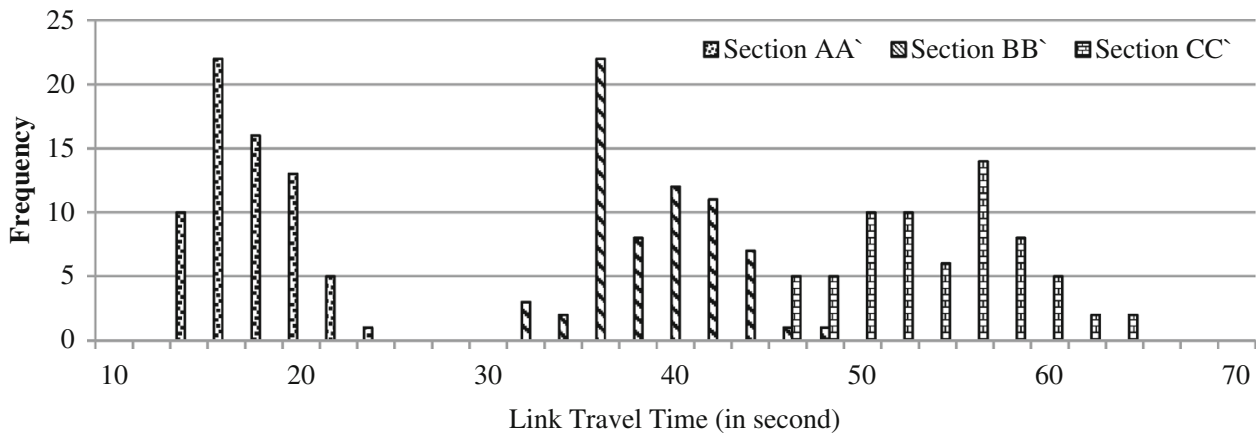

Fig. 11 Distribution of link travel time at different downstream sections
$\alpha=\frac{A_{1} \times e^{\frac{A_{2}}{t_{\text {mean }}}} \times n^{-A_{3}}}{1+\ln \left[\left(\% V G I \times A_{4}+\% V G I I \times A_{5}\right) \times\left(\frac{v}{s}\right)\right]}$

with $\alpha$. Figure 4 indicates that with the increase in link travel time $\alpha$ decreases, as found for different downstream sections (viz. $\mathrm{AA}^{\prime}, \mathrm{BB}^{\prime}$ and $\mathrm{CC}^{\prime}$ ). Similar trend is also observed for modelling step size $(n)$ with $\alpha$ at each downstream section (from Fig. 4). Accordingly, formulation of $\left(1 / t_{\text {mean }}\right)$ and ' $n$ ' with $\alpha$ with functions, which indicates reciprocal relation, were investigated and finally, exponential function and negative power functions were found to be suitable for $\left(1 / \mathrm{t}_{\text {mean }}\right)$ and ' $n$ ' respectively. The empirical relation of 'vehicle group' $(V G)$ and 'normalised flow' $(v / s)$ with $\alpha$ were found complex in nature. The variation of $\alpha$ with ' $V G$ ' and ' $v / s$ ' do not reflect any specific trend which may be modelled by widely used functions such as power functions, exponential functions, logarithmic functions etc. Therefore, previous works $[33,34]$ dealing with the empirical relation of 'vehicle composition' and 'volume to capacity' ratio with macroscopic traffic state parameters such speed, flow and density were studied to get a preliminary idea about the model formulation. To start with, formulation proposed by Maitra et al. [33] to model the effects of 'vehicle composition' and 'volume to capacity'ratio on 'vehicular stream speed' at mid-block location was studied and improvised further to model the combined effects of 'vehicle group' and 'normalised flow' on $\alpha$. Final specification of the proposed empirical model is given shown in Eq. 10. where, $A_{1}, A_{2}, A_{3}, A_{4}$ and $A_{5}$ are the coefficients of this model. Sum of squared errors between calibrated $\alpha$ and model output is minimised to get the values of coefficients. $\% V G-I I I$ is excluded from the model formulation as $\% V G-I$ and $\% V G-I I$ are sufficient enough to indirectly reflect the overall vehicle composition in the traffic stream. Values of the coefficients are found as $A_{1}=0.87$, $A_{2}=8.66, \quad A_{3}=0.77, \quad \mathrm{~A}_{4}=27.04$ and $A_{5}=10.01$ respectively. Regression coefficient of the proposed model was found as 0.663 , which is statistically acceptable. F-test and t-test were also carried out to determine whether the best-fit values of $\alpha$ and model output differ significantly or not. Values of $F$ and $t$ are found to be $0.66\left(F_{c}=0.86\right)$ and $0.98\left(\mathrm{t}_{\mathrm{c}}=1.97\right)$ respectively which imply that at $5 \%$ significance level model outputs are not statistically significantly different from calibrated values of $\alpha$. A comparison between the calibrated dispersion factor $\left(\alpha_{\mathrm{RM}^{-}}\right.$ using Robertson's model) and the modelled dispersion factor ( $\alpha_{E M}$-using empirical model) is shown in Fig. 12.

Equation 10 depicts that with fixed 'normalised flow' and vehicle composition, $\alpha$ is a decreasing function of link travel time. A sample trend is shown in Fig. 13 for modelling step size 3 . Figure 13 demonstrate the variation of $\alpha$ 
Fig. 12 Variation of $\alpha_{\mathrm{RM}}$ and $\alpha_{\mathrm{EM}}$ with different modelling step size

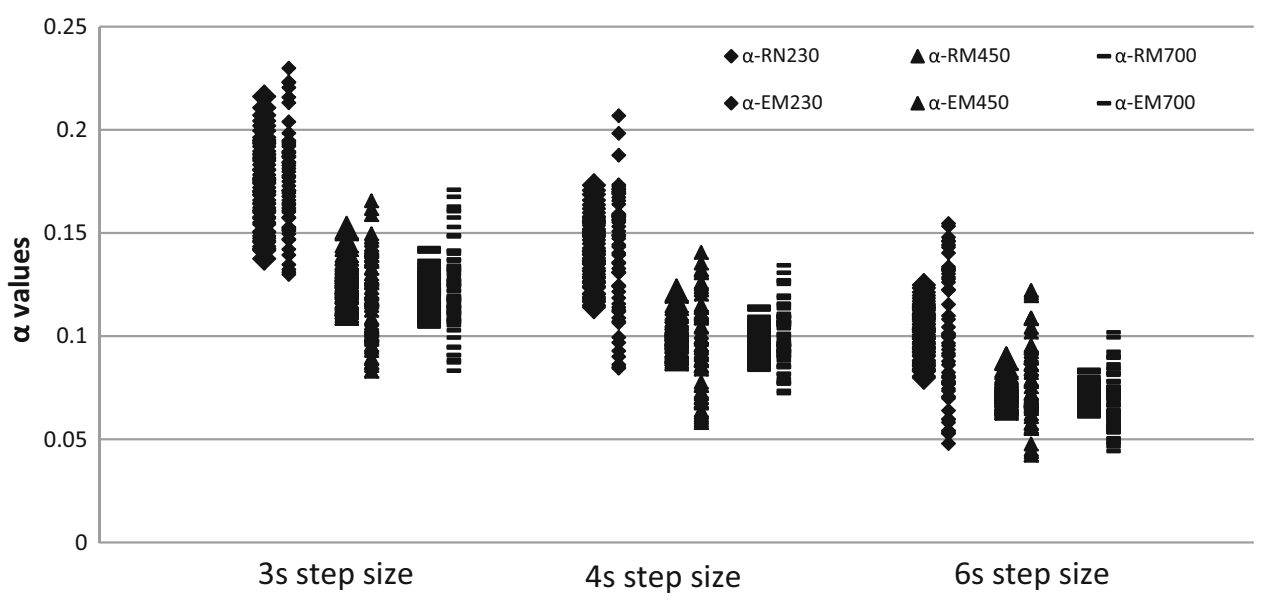

Fig. 13 Platoon dispersion factor as a function of vehicle group, normalised flow, and link travel time

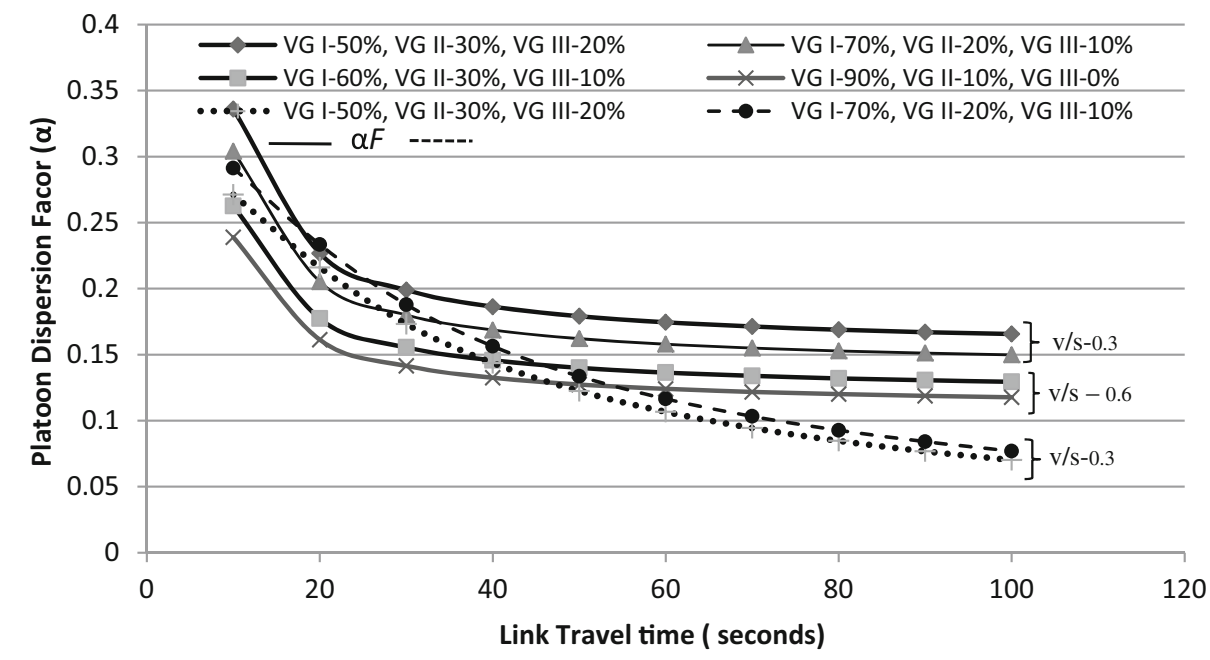

with several traffic state parameters (viz. link length, vehicle composition, normalised flow). Figure 13 represents a decrease in $\alpha$ with the increase in share of car $\left(V G^{-}\right.$ $I)$ for a given set of link travel time $\left(\mathrm{t}_{\text {mean }}\right)$ and normalised flow $(v / s)$ which is quite rationale for a mixed vehicle stream. Platoon dispersion factor $(\alpha)$ also decreases with an increase in normalised flow $(v / s)$ as can be seen in Fig. 13. Manar and Bass [14] and Bie et al. [5] also reported a decreasing trend of $\alpha$ with an increase in 'volume to capacity ratio' (in the range from 0.70 to 1.00 ), which corroborate findings from this study. Variation of smoothening factor $(\mathrm{F})$ with link travel time is also given in Fig. 13 for 'normalised flow' of 0.3. it may be noted from Eq. 1, that $\mathrm{F}$ (smoothening factor) is the primary parameter used to predicts the downstream arrivals for a given discharges from the upstream stop-line. In Robertson's model [8], the smoothening factor $F$ is expressed in terms of two empirical parameters $\alpha$ and $\beta$, which capture the effect of observed traffic state and roadway environment. In course of minimising the sum of squared errors between observed and predicted arrivals, $\mathrm{F}$ is optimised, which is further used to estimate $\alpha$ with a fixed value of $\beta(=0.80)$ using Eq. 2 . It can be seen from Fig. 12 that $\mathrm{F}$ changes inversely with $\mathrm{t}_{\text {mean }}$, i.e. $\mathrm{F}$ decreases with increase in travel time or in other words, vehicle platoons experience more dispersion towards downstream direction. This trend is quite rational and also reported by $\mathrm{Yu}$ [7].

Now, considering Eq. 2 , change in $\mathrm{F}$ i.e. $\Delta \mathrm{F}$ occurs due to change in the product of $\alpha$ and $\mathrm{Ta}\left(\Delta\left(\alpha \mathrm{T}_{\mathrm{a}}\right)\right)$ as $\beta$ is assumed to be fixed as 0.80 . When $\Delta \mathrm{F}$ is negative, $\Delta\left(\alpha \mathrm{T}_{\mathrm{a}}\right)$ is positive which may be obtained from one of the following scenarios, such as (1) increase in both $T_{a}$ and $\alpha$ or (2) increase in $T_{a}$ but decrease in $\alpha$. In later case, scale of increase in Ta may be large enough to result an increase $\Delta(\alpha \mathrm{Ta})$ even with a decrease in $\alpha$ (which eventually results negative $\Delta \mathrm{F}$ ). Based on the data collected at the field, both scenarios (1) and (2) were observed. However, scenario (2) was observed to be predominant, leading to the proposed empirical model indicating an inverse trend of $\alpha$ with $T_{a}$, which does not violate the underlying phenomenon of 
vehicle dispersion as expressed by Robertson's model. Similar observations were also reported by Rakha and Farzaneh [16].

Based on all these findings it may be concluded that proposed empirical model is suitable for urban traffic corridor in emerging countries, where vehicle mix is predominantly heterogenous. To develop time of day signal timing plans, the model will be able to capture the effect of variation in travel demand and vehicle composition. For an instance, during the period of lower normalized flow ( $\mathrm{v} /$ $\mathrm{s}=0.3$ ) for a longer link (with mean travel time $80 \mathrm{~s}$ to $100 \mathrm{~s}$ ), high value of $\alpha$ (in the range of 0.15 to 0.17 ) is more suitable. Similarly, at a level of 'v/s' as 0.60 , higher share of car in a vehicle mix (i.e., 0.9 or more) require relatively low value of $\alpha$ (in the range of 0.12 to 0.13 ) than a lower share of car in it. Another significant revelation by the model is that for shorter links (viz. mean travel time $20 \mathrm{~s}$ or less), variation in vehicle mix has larger effect on platoon dispersion than longer links.

\section{Conclusion}

The present study brings several interesting findings related to the calibration of Robertson's platoon dispersion model in a typical urban scenario with heterogenous traffic and non-labe based traffic operation. With a fixed value of travel time factor $(\beta)$ as 0.80 , the platoon dispersion factor $(\alpha)$ is calibrated and found to vary depending on the distance covered by vehicle platoon from upstream stop-line. $\alpha$ is also found to vary based on the traffic cmposition and size of modelling step.A major contribution from the present work is development of a generalised model which may be used advantageously to obtain a suitable $\alpha$ giving due consideration to prevailing traffic state parameters (viz. vehicle group, normalised flow at stop-line), network parameters (viz. link length) and parameter of model state (viz. size of modelling step) simultaneously. The findings reported in the paper are case specific, but are expected to provide an insight on platoon dispersion of urban corridors operating under non-lane based mixed traffic condition. The coefficients of the proposed generalised model are needed to be validated based on further field investigation in order to judge the geographical transferability of the model under different traffic corridors and vehicle compositional distribution.

\section{References}

1. Roess RP, Prassas ES, McShane WR (2010) Traffic engineering. Pearson Prentice Hall, New Jersey
2. Highway capacity manual (2000) Transportation Research Board. National Research Council, Washington, D.C.

3. Rouphail N, Tarko A, Li J (1992) Traffic flow at signalized intersections, revised monograph on traffic flow theory, chapter 9

4. Jiang Y, Li S, Shamoc DE (2006) A platoon-based traffic signal timing algorithm for major-minor intersection types. Transp Res Part B Methodol 40(7):543-562

5. Bie Y, Liu Z, Ma D, Wang D (2013) Calibration of platoon dispersion parameters considering the impact of number of lanes. J Transp Eng 139:200-207

6. Farzaneh M (205) Modelling traffic dispersion. PhD Thesis, Department of Civil Engineering, Virginia Polytechnic Institute and State University

7. Yu L (2000) Calibration of platoon dispersion parameters on the basis of link travel time statistics, Transportation Research Record, vol 1727. Transportation Research Board, Washington, D.C., pp 89-94

8. Denney RW (1989) Traffic platoon dispersion modelling. J Transp Eng 115:193-207

9. Tarnof PJ, Parsonson PS (1981) Guidelines for selecting traffic signal control at individual intersections, NCHRP report no. 233

10. Chang EC-P, Messer CJ (1986) Warrants for interconnection of isolated traffic signals. Federal Highway Administration, Washington, D.C.

11. Qiao F, Yang H, Lam WHK (2001) Intelligent simulation and prediction of traffic flow dispersion. Transp Res Part B 35:843-863

12. Robertson DI (1969) TRANSYT: a traffic network study tool, Road Research Laboratory Report, LR 253. Road Research Laboratory, Crowthorne

13. Seddon PA (1972) Another look at platoon dispersion: 3. the recurrence relationship. Traffic Eng Control 13(10):442-444

14. Manar A, Bass K (1996) Traffic platoon dispersion modelling on arterial streets, Transportation Research Record, vol 1566. Transportation Research Board, Washington, D.C.

15. Bass KG, Lefebvre S (1988) Analysis of platoon dispersion with respect to traffic volume, Transportation Research Record, vol 1194. Transportation Research Board, Washington, D.C.

16. Rakha H, Farzaneh M (2005) Macroscopic modelling of traffic dispersion: Issues and proposed solutions, $84^{\text {th }}$ Transportation Research Board Annual Meeting, Washington, D.C.

17. Farzaneh M, Rakha H (2006) Procedures for calibrating TRANSYT platoon dispersion model. J Transp Eng 132(7):548-554

18. El-Reedy TY, Ashworth R (1978) Platoon dispersion along a major road in Sheffild. Traffic Eng Control 19(4):186-189

19. Arasan VT, Kashani SH (2005) Modelling platoon dispersal pattern of heterogeneous road traffic, 82nd Transportation Research Board Annual Meeting, Washington, D.C.

20. He Q, Head KL, Ding J (2012) Platoon-based arterial multimodal signal control with online data, Transp Res Part C, pp 164-184

21. Lighthill MJ, Whitham GB (1955) On kinematic waves: II. A theory of traffic flow on long crowded roads. Proc R Soc Lond Ser A 229:317-345

22. Pacey GM (1956) The progress of a bunch of vehicles released from a traffic signal, Research Note Rn/2665/GMP. Road Research Laboratory, London

23. May AD (1990) Traffic flow fundamentals. Prentice Hall, New Jersey

24. Geroliminis N, Skabardonis A (2005) Prediction of arrival profiles and queue lengths along signalized arterials by using a markov decision process. Transp Res Rec J Transp Res Board, No. 1934, Transportation Research Board of the National Academies, Washington, D.C., pp 116-124 
25. Hunt PB, Robertson DI, Bretherton RD, Winton RI (1981) SCOOT - a traffic responsive method of coordinating signals, RRL Report LR 1041. Road Research Laboratory, UK

26. Hall MD, Van Vliet D, Willumsen LG (1980) SATURN-a simulation/assignment model for the evaluation of traffic management schemes. Traffic Eng Control 21(4):168-176

27. Lieberman EB, Andrews BJ (1980) TRAFLO-a new tool to evaluate transportation management strategies. Transp Res Rec 772:9-15

28. Seddon PA (1972) The prediction of platoon dispersion in the combination methods of linking traffic signal. Transp Res 6(2): $125-130$

29. Guebert AA, Sparks G (1989) Timing plan sensitivity to changes in platoon setting. University of Saskatoon, Saskatoon

30. Yu L, Van Aerde M (1995) Implementing TRANSYT's macroscopic platoon dispersion in microscopic traffic simulation models, 74th Transportation Research Board Annual Meeting, Washington, D.C.

31. Athol P (1965) Headway groupings, Highway research record 72. HRB, National Research Council, Washington, D.C, pp 137-155

32. Special publication 41 (1994) Guidelines for the design of atgrade intersections in rural and urban areas, The Indian Road Congress, New Delhi

33. Maitra B, Sikdar PK, Dhingra SL (1999) Modeling congestion on urban roads and assessing level of service. J Transp Eng 125-6:508-514

34. Velmurugan S, Madhu E, Ravinder K, Sitaramanjaneyulu K, Gangopadhyay S (2010) Critical evaluation of roadway capacity of multi-lane high speed corridors under heterogeneous traffic conditions through traditional and microscopic simulation models. J Indian Roads Congr 566:235-264 